Cahiers d'études africaines

$240 \mid 2020$

Soudan : identités en tension

\title{
Ethnicité, religion, nationalisme
}

Intersections et ambiguïtés dans un Soudan en mouvement

Ethnicity, Religion, Nationalism. Intersections and Ambiguities in a Shifting Sudan

Barbara Casciarri, Alice Franck, Stefano Manfredi et Munzoul Assal

\section{OpenEdition}

\section{Journals}

Édition électronique

URL : https://journals.openedition.org/etudesafricaines/32172

DOI : 10.4000 /etudesafricaines.32172

ISSN : $1777-5353$

Éditeur

Éditions de l'EHESS

\section{Édition imprimée}

Date de publication : 2 décembre 2020

Pagination : 761-778

ISSN : 0008-0055

Référence électronique

Barbara Casciarri, Alice Franck, Stefano Manfredi et Munzoul Assal, «Ethnicité, religion,

nationalisme », Cahiers d'études africaines [En ligne], 240 | 2020, mis en ligne le 02 décembre 2020,

consulté le 05 janvier 2023. URL : http://journals.openedition.org/etudesafricaines/32172 ; DOI :

https://doi.org/10.4000/etudesafricaines.32172 
Barbara Casciarri, Alice Franck, Stefano Manfredi \& Munzoul Assal

\title{
Ethnicité, religion, nationalisme
}

\author{
Intersections et ambiguïtés \\ dans un Soudan en mouvement*
}

Au Soudan, les notions d'arabité et d'islamité sont au cœur des critères définissant les inscriptions identitaires qui affectent encore largement les dynamiques locales. Ces deux notions, sources de tension jusqu'à nos jours, se conjuguent avec la notion d'identité nationale, ou « soudanité » dont la construction, rendue complexe par l'héritage colonial, a débouché sur de nombreuses impasses en phase postcoloniale. Ces trois notions sont au centre d'enjeux cruciaux, en termes de classements statutaires, de mécanismes d'exclusion ou d'inclusion. Elles ont été mobilisées — diversement mais constamment - dans le cadre des conflits meurtriers ayant affecté le pays depuis l'indépendance. Pourtant, des ambiguïtés demeurent dans leur catégorisation et leur articulation, nuisant à la compréhension des processus qui y sont liés. La conjoncture politique récente a mis en exergue la centralité de cette problématique : au niveau international, on assiste à l'émergence d'un intérêt renouvelé vis-à-vis des enjeux liés à l'islamité et à l'arabité dans un cadre hautement conflictuel, exemplifié par les crises et interventions militaires au Moyen-Orient et en Afrique, et leurs résonances en Europe (attentats ou jihad islamiques, échos du « clash de civilisations » islam/Occident, islamophobie). Au niveau national, la longue guerre civile entre le Nord et le Sud (1955-1972; 1983-2005), au cours de laquelle la polarisation par l'ethnicisation du conflit a été utilisée par les deux parties, a finalement débouché sur l'échec d'une construction nationale qui s'est matérialisé par la séparation du Soudan du Sud

* En plus des institutions qui ont financé le projet de recherche à l'origine de ce numéro (AUF, CEDEJ, Université Paris 8, LAVUE, PRODIG, SeDyL), nous remercions aussi les relecteurs des articles et tous les chercheurs qui, ayant collaboré au sein du programme AUF, n'ont pas pu présenter leurs contributions dans ce numéro, mais le feront par la suite dans un ouvrage collectif en gestation. 
en 2011. La création du nouvel État, loin de clore les ambiguïtés, a ouvert un nouvel espace à la redéfinition et à la réarticulation de ces identités au cœur des relations sociopolitiques du pays, faisant du Soudan un laboratoire idéal pour une étude des (re)configurations identitaires. Les mobilisations d'ampleur nationale qui ont démarré en décembre 2018 pour aboutir en avril 2019 à la chute du régime islamique en place depuis 1989 confirment la nécessité de développer à partir du cas soudanais une réflexion interdisciplinaire sur les manières dont les appartenances, les pratiques et les politiques touchant aux trois catégories (arabité, islamité et soudanité) ${ }^{1} \mathrm{~s}^{\prime}$ imbriquent et se donnent à voir dans des processus sociaux concrets ${ }^{2}$.

Les notions d'ethnicité, de religion et de nationalisme recouvrent des domaines de la réalité sociale (au Soudan et ailleurs) aussi amples et variés que les espaces pluridisciplinaires des débats scientifiques qui les ont analysées. Nous avons choisi de les interroger au travers des trois déclinaisons — arabité, islamité et « soudanité » — que l'histoire et les dynamiques sociales du Soudan ont fait émerger comme pertinentes. Si l'arabité est loin de résumer la pluralité des appartenances ethniques locales, elle s'est néanmoins imposée progressivement comme un référent majeur par rapport auquel tout individu ou groupe est censé se positionner, conduisant à l'inclusion ou au contraire à la marginalisation dans un statut de «minorité ». L'islamité témoigne d'un parcours analogue. À partir de l'arrivée au Soudan de populations conquérantes venues d'Arabie saoudite et d'Égypte (VII siècle), puis avec le sultanat de Sennar (1506-1821), première formation étatique à se revendiquer comme musulmane, l'islamité s'inscrit dans l'identité soudanaise et pose les bases d'une assimilation mécanique à l'identité arabe. La centralité des débats politiques depuis la phase d'indépendance autour de la définition du Soudan comme «pays arabo-musulman » atteste cette relation ambiguë. Jusqu'à nos jours, l'affiliation à l'islam dépasse largement la dimension de croyance religieuse, établissant une ligne de démarcation en matière statutaire et conditionnant le plein accès aux droits, aux ressources matérielles et à la citoyenneté.

1. Ce numéro s'appuie sur le travail réalisé par une équipe interdisciplinaire et internationale dans le cadre du projet AUF-PCSI (2018-2020) Arabité, islamité, "soudanité ». Enjeux de la redéfinition des appartenances, des pratiques locales et des politiques étatiques au Soudan après la séparation du Sud-Soudan (CEDEJ, University of Khartoum, Université Paris 8/LAVUE, Max Planck Institute).

2. En ce qui concerne la transcription des termes arabes, nous avons opté pour la solution suivante : pour les textes où les auteurs se basent sur des sources écrites, nous avons laissé leur choix d'une transcription traditionnelle de l'arabe classique et standard. Pour les textes où ces termes se réfèrent à des réalités de terrain et à des sources orales, nous avons préféré adopter une transcription phonologique de l'arabe soudanais. Ce choix s'inscrit en cohérence avec la problématique de ce numéro, qui souhaite éviter le risque d'homogénéiser et hiérarchiser ces diversités intrinsèques de la langue arabe. 
CARTE 1. - CARTE ADMINISTRATIVE DU SOUdAN APRÈS 2011
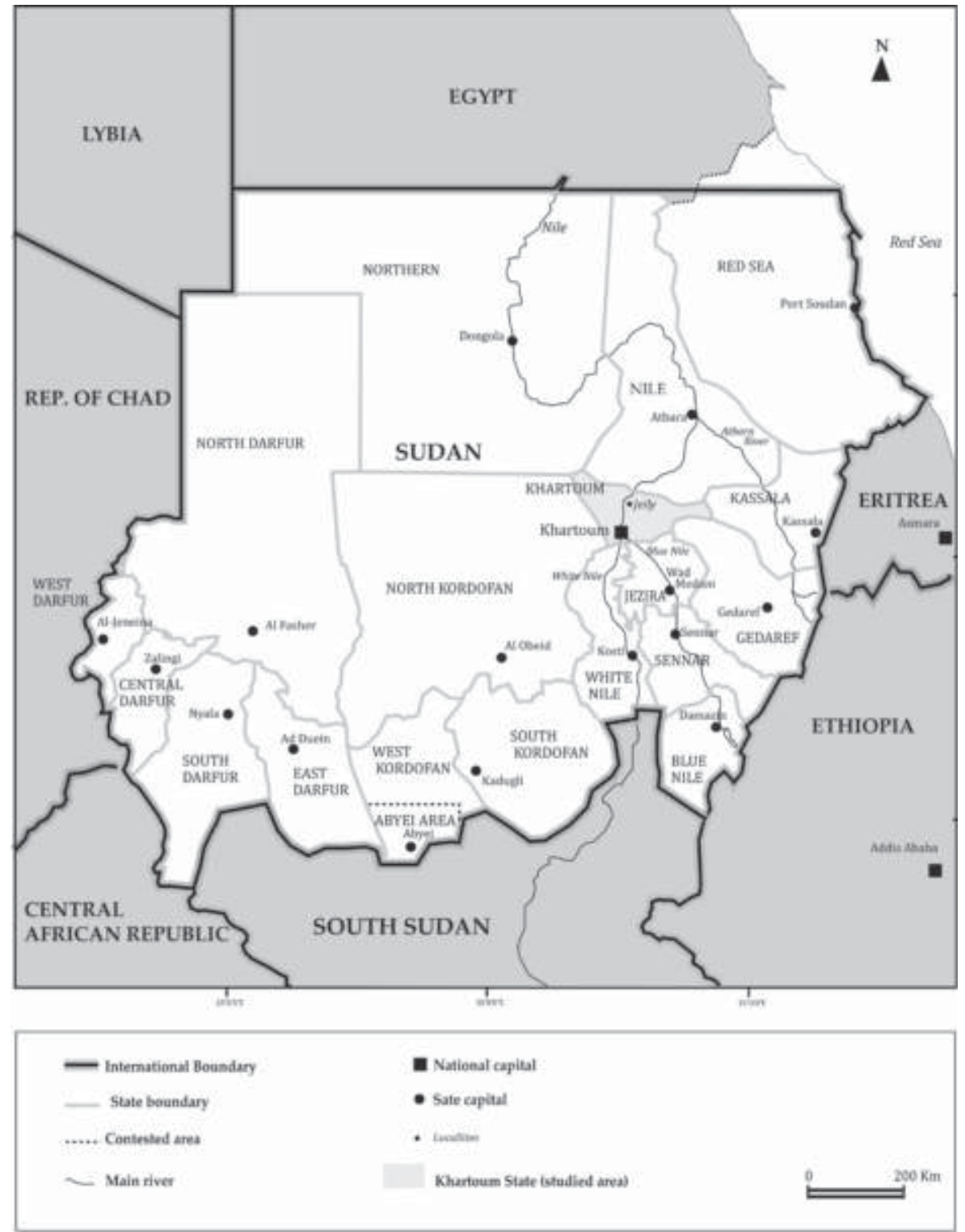

Source : Université du Texas, <http://www.lib.utexas.edu/maps/africa/txu-oclc-224306541-sudan_ darfur_2007.jpg >. Open Street map. Réalisation A. Franck, 2020. 
La définition du « Soudan » restreinte à l'État-nation s'est affirmée bien plus tardivement ( $\mathrm{XIX}^{\mathrm{e}}-\mathrm{XX}^{\mathrm{e}}$ siècles) par rapport à l'acception à l'origine élargie à l'ensemble de la bande sahélienne (très largement corrélée à cette assimilation entre arabité et islamité), qui était celle des géographes arabes puis des colonisateurs. Pourtant, la construction d'une identité nationale en phase postcoloniale est restée strictement tributaire de son association à ce double référent de l'identité des Soudanais en tant qu'arabes et musulmans. Les études soudanaises doivent ainsi interroger l'imbrication, les agencements historiques et les déclinaisons autour de ces trois notions d'ethnicité, de religion et de nationalisme, ici évoquées comme la «triade» arabité, islamité, soudanité pour appréhender en profondeur la question des appartenances et de leur évolution. Consacré aux reconfigurations identitaires et ancré dans des enquêtes ethnographiques, ce numéro souhaite contribuer à l'analyse dynamique des intersections et ambiguités persistantes au sein de ce que nous avons appelé le " complexe identitaire soudanais », recoupant parfois les notions de race, de tribu ou encore de classe, en sollicitant le débat théorique des sciences sociales et en ouvrant sur la comparaison au-delà de la spécificité du cas soudanais.

Au-delà du déconstructionisme: anthropologie et identités au Soudan

Le tournant déconstructiviste et anti-essentialiste inauguré par Ethnic Groups and Boundaries (Barth 1969) peut se vanter d'avoir passé l'épreuve du temps en dépit de quelques critiques (Hummel 2014), mais également d'avoir impulsé un élargissement de sa démarche théorique à des espaces d'analyse de la construction identitaire qui dépassent l'ethnicité au sens strict. L'apport historique des travaux de Fredrik Barth et de son école pour l'analyse des processus de construction de l'ethnicité a été particulièrement significatif pour le contexte soudanais - où l'anthropologue avait d'ailleurs mené un de ses terrains ainsi que certains de ses disciples qui ont par la suite marqué les études soudanaises (Haaland 1969, 2014 ; Ahmed 2002). Inspirés par cette approche fondatrice, les chercheurs se sont par la suite penchés sur les dynamiques occultées du discours identitaire, en proposant deux cadres analytiques prioritaires. Le premier insiste sur le rôle des élites dominantes dans la fixation de catégories dichotomiques ainsi que sur celui de l'État dans leur hiérarchisation pour asseoir la domination du «centre» sur ses «périphéries» (Harir \& Tvedt 1994) : ces travaux voient dans ce processus la matrice de violences historiques et d'un «désordre durable » (Sorbo \& Ahmed 2013), dont l'explication en termes de conflits identitaires, ethniques ou tribaux, relèverait d'une dissimulation idéologique. Le second 
cadre analytique dévoile l'imbrication étroite entre, d'une part, division du travail et formation de classes et, d'autre part, dynamiques de l'ethnicité (O'Brien 1986) : de l'instauration d'un système capitaliste en phase coloniale jusqu'au consolidement d'un développement inégal persistant, c'est plutôt dans les injustices de l'accès aux ressources et dans la compétition pour l'exploitation des territoires (Assal 2006) que se noueraient les reconfigurations des appartenances et leurs logiques de domination. Corollaire de ces deux cadres analytiques majeurs, l'expérience coloniale a été reconnue comme moment clé de la configuration ambiguë de ce complexe identitaire. En termes plus généraux, la formation de l'État moderne a été décryptée comme intrinsèquement portée par l'exigence de catégoriser pour gouverner, selon des procédures de «lisibilité » et de «simplification 》 (Scott 1998) investissant autant les ressources naturelles que les communautés humaines. Plus spécifiquement, les politiques de gouvernement indirect de la colonisation britannique au Soudan, de par leurs mécanismes d'administration et de «tribalisation » des populations soudanaises (Grandin 1982), ont été lues comme fondatrices de la construction du native en tant qu'identité politique (Mamdani 2012).

Tout en nous inscrivant dans la continuité de ces analyses, plusieurs éléments poussent à revenir sur le paradigme identitaire posé en termes d'oppositions binaires pour le confronter avec une réalité soudanaise en mouvement. De fait, si l'anthropologie et d'autres sciences sociales ont continué à décrypter les dynamiques créatives d'identification collective - et leurs manipulations politiques (Schlee \& Watson 2009) —, elles n'ont pas toujours mené jusqu'au bout l'impulsion des approches pionnières de la démarche anti-essentialiste. Ceci s'illustre par exemple dans la cristallisation d'une synthèse de l'histoire du Soudan préfigurant sa configuration actuelle autour de l'arrivée, à partir des premiers siècles d'expansion de l'islam, de populations arabes et musulmanes (principalement nomades) venant de la péninsule arabique et d'Égypte, qui se seraient superposées aux groupes autochtones pour en déterminer progressivement l'arabisation et l'islamisation. Véhiculée à la fois par des historiens coloniaux (MacMichael 1922; Trimingham 1949) et postcoloniaux (Fadl Hasan 1967), confortée par les récits des groupes locaux, cette version a été également reprise dans ses grandes lignes par les travaux des chercheurs en sciences sociales. Tandis que ces derniers n'hésitent pas à évoquer la prégnance de la « diversité ethnique » et du " pluralisme culturel », ainsi qu'à contester la dichotomisation des composantes « arabe » et « africaine» du pays (Harir 1994), force est de constater les quelques paradoxes de cette vulgate. D'abord, le caractère presque «naturel » qu'elle semble suggérer de ces vagues de changement socioculturel complexe (Manger 1994); puis, la 
réification et l'homogénéisation de deux catégories (arabité et islamité) dont la définition et l'articulation ne vont pas de soi (Casciarri 2018); enfin, le manque de clarté sur l'imbrication mouvante des concepts d'ethnicité, identité et nationalisme (Manger 2012). L'usage récurrent dans les études soudanaises de certaines notions — « arabisation » ou « islamisation » (Fluher-Lobban 1987; Delmet 1991), « soudanisation » ou encore « mode de vie arabo-musulman » (Doornbos 1998) - semble révéler les risques d'un retour vers les pièges essentialistes, en ce qu'il conforte des images persistantes d'une supposée présence de cultures autochtones originales, authentiques et isolées.

Les articles de ce numéro proposent différents cas d'étude qui rouvrent le débat critique sur le "complexe identitaire» soudanais et tentent de le dépasser en mobilisant à la fois des données empiriques inédites et des angles de lecture originaux, tout en s'appuyant sur les contributions précédemment citées. Si le paradigme anti-essentialiste, hanté par les spectres du racisme et du déterminisme biologique, a détourné le regard des chercheurs des expressions physiques de l'altérité, l'anthropologue Azza Ahmed Abdel Aziz centre son texte sur les conceptualisations courantes des couleurs de peau : son analyse du discours, d'acteurs locaux comme de chercheurs, sur cet objet refoulé permet non seulement de saisir l'héritage des rapports d'esclavage sur la construction des hiérarchies ethniques et sociales, historiques et contemporaines, mais également de plonger dans la subtilité d'une particularité identitaire qui est vécue et incorporée par les sujets pour ensuite gagner une dimension intersubjective par la circulation des récits. D'autres postulats de la tradition déconstructiviste sont aussi rouverts au questionnement. En retraçant le traitement de la diversité ethnique et tribale 3 à l'époque mahdiste (1881-1898), notamment par l'ethnicisation ambiguë des groupes beja dans l'Est soudanais, l'historien Anaël Poussier montre que certaines logiques de réification et de manipulation des identités locales ont pu caractériser l'action d'un État précolonial : si la colonisation a donc été un moment crucial de la catégorisation binaire et hiérarchique des identités (Mamdani 2012), la tendance étatique à essentialiser pour mieux gouverner relève d'une histoire plus ancienne. Dans un tout autre contexte et sur une période actuelle, l'article de Philippe Gout, juriste, propose un cas d'étude original : son ethnographie des tribunaux du

3. Dans une acception qui apparaît proche des catégorisations en vigueur auprès des groupes soudanais, et cela malgré la fluidité des démarcations, les deux termes, " ethnique » et «tribal », sont associés mais partiellement distincts : « ethnie» (traduisant plus souvent le terme jins) ferait référence à une dimension plus large de partage de caractéristiques culturelles, linguistiques, d'origines communes présumées, tandis que « tribu » (traduisant plus univoquement le terme gabila) indique un groupe aux dimensions plus réduites, qui associe à l'idée de filiation unilinéaire partagée des prérogatives politiques et territoriales (CASCIARRI 2016). 
droit d'asile en France nous porte à découvrir un autre acteur inattendu des politiques de construction identitaire, l'OFPRA (Office français de protection des réfugiés et apatrides). En dépit de sa prétendue objectivité, gage de l'expression d'une « communauté internationale » et de la modernité de ses lois, cette institution apparaît non seulement prise dans les pièges essentialistes mais semble aussi contribuer fortement par ses jugements à confirmer et renforcer l'ambivalence identitaire et la binarité des catégorisations des « Soudanais ». C'est encore un anthropologue, Mohamed Bakhit, qui, par un retour sur la place des périphéries urbaines et des populations y résidant lors de la révolution de 2019, brise un autre lieu commun, celui de la superposition des dichotomies « ville $v s$ campagne » et « universalisme moderne vs particularisme archaïque », en illustrant l'enracinement des assignations identitaires (ethniques, tribales, raciales) dans la capitale, creuset d'un cosmopolitisme fantasmé. Enfin, deux jeunes anthropologues soudanais présentent via leurs notes de recherches en cours, deux autres cas emblématiques de la complexité des déclinaisons identitaires au Soudan contemporain: Hager Hassan illustre comment les Seleim, pasteurs du Nil Blanc, ont construit une «identité duale », capable de transcender les oppositions Arabes/Africains, musulmans/chrétiens, et même nomades/sédentaires, aujourd'hui remise en question par les événements dramatiques ayant suivi la séparation du Soudan du Sud. Abdeljbar Ejami propose quant à lui une critique de la lecture ethnique et tribale des conflits récents dans l'Est Soudan, en dévoilant les enjeux sous-jacents de la relation entre élites politiques et religieuses locales et de l'héritage colonial d'un système d'exploitation capitaliste. Ainsi, un demi-siècle après l'émergence $\mathrm{du}$ « tournant anti-essentialiste» dans le domaine des questions identitaires, l'anthropologie continue d'occuper une place de choix dans ce débat, mais elle s'est renouvelée par les apports d'autres perspectives disciplinaires et de nouveaux cas d'étude témoignant de nouvelles reconfigurations.

\section{Langues et éducation: entre arabisation et pluralisme}

L'imbrication entre la question linguistique et les processus ou politiques d'éducation constitue un autre espace pertinent pour appréhender et suivre l'articulation des catégorisations identitaires. Avec ses 77 langues appartenant à trois familles linguistiques différentes (Eberhard et al. 2020), le Soudan est un pays extrêmement hétérogène sur le plan ethnolinguistique. Depuis l'indépendance du pays, cette diversité a représenté un enjeu majeur pour la construction d'une identité nationale partagée ainsi que pour la formalisation des politiques éducatives qui se sont succédé durant la période postcoloniale. 
Les accords de paix de Naivasha (CPA) ${ }^{4}$ en 2005 (Abdelhay et al. 2011) ont formellement mis un terme à des décennies d'arabisation linguistique et culturelle dont les conséquences en termes de catégories d'appartenance des communautés locales ont été démontrées par la centralité de la composante ethnolinguistique dans une grande partie des crises régionales ayant traversé le Soudan (principalement au Soudan du Sud et dans les Monts Nouba). Pourtant, cette réorientation pluraliste post-2005 qui visait, entre autres, à intégrer les langues dites « indigènes » dans l'éducation primaire (Abdelhay et al. 2015), n'a pas produit d'effets tangibles en termes de reconnaissance et de développement des langues minoritaires du Soudan. En revanche, étant largement inspirée par une vision coloniale et ethnicisante de la diversité linguistique, cette politique a largement contribué à la fixation des frontières tribales $^{5}$ et, dans certains cas, à l'émergence de nouveaux conflits de type ethnolinguistique (Manfredi 2015). Après l'indépendance du Soudan du Sud en 2011, la politique multilingue entreprise par le CPA n'a pas été officiellement abandonnée, cependant l'arabe reste, de facto, la seule langue officielle et nationale du Soudan. À ce propos, il est intéressant de souligner le caractère monolingue de la révolution soudanaise de 2018-2019 qui, malgré son orientation transversale et inclusive, reste un phénomène largement arabophone, loin des revendications de type ethnolinguistique qui auraient pu créer des fragmentations communautaristes au sein du mouvement (Casciarri \& Manfredi 2020). Malgré cela, la définition du statut et des fonctions des différentes langues en concurrence dans l'espace public sera un élément fondamental à prendre en compte dans la mise en place des nouvelles politiques éducatives visant à réduire les inégalités ethnoculturelles dans le Soudan postrévolutionnaire.

De fait, la langue arabe a toujours joué un rôle fondamental et controversé dans la construction identitaire du Soudan postcolonial. De plus, l'association entre langue, ethnicité et religion, qui trouve son origine dans une lecture simpliste et dichotomique de l'histoire soudanaise (Arabes vs Africains), a été largement employée comme instrument de manipulation idéologique par le régime islamiste d'Omar Al-Bechir entre 1989 et 2019 (Sharkey 2007). Ayant été forgée entre la fin du condominium anglo-égyptien et la proclamation

4. CPA désigne le Comprehensive Peace Agreement, accords de paix mettant fin à la plus longue des guerres civiles soudanaises, celle entre Nord et Sud (démarrée en 1955 et interrompue uniquement, entre 1972 et 1983, par la trêve établie lors des Accords d'Addis-Abeba). Le CPA prévoyait six années intérimaires d'un gouvernement d'unité nationale, devant se clôturer en 2011 par un référendum d'autodétermination du Sud censé déterminer la séparation du Sud ou le maintien de l'unité du Soudan.

5. L'article de Philippe Gout, dans ce numéro, revient sur le rôle que joue la langue dans l'identification ethnique dans un contexte non soudanais et éclaire, dans le même temps, la nécessité de décoloniser les institutions judiciaires du droit d'asile. 
d'indépendance du Soudan, l'articulation idéologique entre arabité, islamité et identité nationale ne peut être considérée comme un effet politique induit par la décolonisation du Soudan. Comme le dévoilent deux contributions au présent numéro, l'arabisation et l'islamisation sont plutôt des constantes politiques indépendantes, mais complémentaires, qui ont été déclinées sous différentes formes dans diverses phases de l'histoire du Soudan. Dans son article, Iris SeriHersch questionne la création d'un système éducatif postcolonial au Soudan méridional en situant le début des politiques d'arabisation et d'islamisation à la fin de l'époque coloniale. Elle examine dans quelle mesure l'adoption de la langue arabe et de la religion islamique a été instrumentalisée par le discours officiel et les politiques de scolarisation entre 1946 et 1964. En outre, elle soutient que les changements politiques qui ont suivi l'indépendance de 1956 ne constituent pas une rupture nette par rapport au passé colonial, mais plutôt une étape du processus de soudanisation culturelle à l'œuvre à partir des années 1940. De manière différente, mais comparable, Heather J. Sharkey montre que la formalisation d'une vision anti-essentialiste et socialement inclusive de la culture arabo-islamique était déjà entamée pendant la première période postcoloniale du Soudan. À travers l'analyse du texte Tarikh al-thaqafa al- 'arabiyy a fi al-Sudan («Histoire de la culture arabe au Soudan»), publié par l'Égyptien 'Abd al-Majid 'Abidin en 1953, Sharkey décrit le milieu intellectuel et académique du jeune État indépendant soudanais, dans lequel s'est forgée l'idée d'une culture arabo-islamique capable de dépasser les différences locales et de construire une identité nationale partagée. Cette ouverture formelle à la diversité ethnoculturelle, qui a caractérisé la démarche intellectuelle des universitaires soudanais juste après l'indépendance, n'a pourtant pas été suffisamment développée pendant les phases successives de la décolonisation. À une autre échelle, Khadidja Medani et Bakheit Nur proposent de resituer l'expérience de l'Université internationale d'Afrique (UIA) à Khartoum dans le processus d'islamisation de la connaissance qui émerge au moment des indépendances des pays arabes, et d'analyser les pratiques pédagogiques et les discours des principaux acteurs institutionnels au sein de cette université. Les auteurs retracent l'évolution des pratiques d'islamisation de la connaissance mises en acte au sein du réseau d'enseignement islamique global. Dans ce contexte particulier, l'adoption de l'arabe comme langue d'enseignement n'est pas une mesure d'assimilation nationale, comme dans le cas de la première époque postcoloniale, mais plutôt un outil qui permet d'unifier l'Umma, entendue comme la communauté islamique transnationale. L'UIA se différencie des autres institutions islamiques par l'accueil d'étudiants étrangers, notamment africains, et par l'enseignement des langues autres que l'arabe (principalement l'anglais et le français, mais aussi le swahili, le hausa, 
le turc et le chinois) qui sont perçues comme de possibles instruments de prosélytisme en Afrique et au-delà. En dépit de la diversité des sujets et des périodes analysés, les trois articles offrent une vision transversale et cohérente sur la complexité de l'association entre arabisation et islamisation au Soudan, et abordent en creux le silence manifeste sur la question de l'ethnicité dans les projets visant à fédérer l'Umma et la nation en devenir.

Vers le croisement des dynamiques de longue et de courte durée

L'exigence de développer aujourd'hui une analyse du cas soudanais capable de brasser des domaines variés (langue, parenté, appropriation de l'espace, accès aux ressources, politique, religieux, droit) qui constituent la toile de fond où se lient et se délient les agencements des trois catégories ethnicité, religion, nationalisme - et dont le corollaire est une incontournable interdisciplinarité - doit s'accompagner d'un effort de contextualisation. La portée des conjonctures historiques apparait, de fait, encore sous-estimée : d'une part, la longue durée a souvent présenté le risque d'être figée dans le retour cyclique d'un passé réduit à l'inconciliable polarisation entre un Soudan africain et un Soudan arabo-musulman (catégories pourtant contestées) (Harir 1994). D'autre part, la dimension événementielle continue d'être privilégiée, avec une attention quasi exclusive portée aux phénomènes macropolitiques, avec un regard souvent façonné par les focalisations éphémères des intérêts d'une géopolitique internationale (Ambrosetti 2011). Pour résoudre ce hiatus, il semble nécessaire de saisir les significations des diverses temporalités qui ont parcouru et parcourent les négociations des auto- et hétéro-définitions des trois catégories ciblées dans leur interaction. À cet égard, l'importance du recours à l'ethnographie pour saisir les agencements des identités ethniques et religieuses des groupes historiquement marginalisés au Soudan « arabomusulman » (Baumann 1987; Manger 2002) a déjà été soulignée, de même que les prémices du nationalisme soudanais au début du $\mathrm{XX}^{\mathrm{e}}$ siècle ont été analysées en lien avec une histoire sociale qui noue relégation raciale et formation de classe (Vezzadini 2015). L'influence des politiques d'administration indirecte dans les reconfigurations tribales et les assises territoriales (Grandin 1982) et celle des politiques linguistiques et d'éducation (Miller 2006; Sharkey 2007; Manfredi 2015 ; Seri-Hersch 2018) ont permis de déceler la complexité des jeux mouvants de construction de ces catégories dans la phase cruciale de la colonisation. C'est en reprenant le fil insuffisamment valorisé de ce type d'analyses, qui croisent dynamiques de longue et de brève durée, que la compréhension des configurations contemporaines de la triade ethnicité-religion-nationalisme au Soudan doit se faire. 
De fait, l'impression d'une accélération inédite et difficile à saisir de l'histoire du Soudan dans la dernière décennie (Casciarri, Assal \& Ireton 2015) s'accompagne d'une attention enthousiaste des recompositions sociales in fieri mais aussi d'une difficulté de sortir d'un présent si dense de transformations. L'arrivée au pouvoir en 1989 d'un régime islamiste — caractérisé comme autoritaire et néolibéral à partir de la moitié des années 1990 — a non seulement marqué le début d'une stabilité gouvernementale inconnue en phase postcoloniale, mais a également balayé les efforts de construction d'une identité nationale « afro-arabe » (Abd Al-Rahim 1971) pour ce pays à la « marginalité multiple » (Mazrui 1971). La période intérimaire du CPA (2005-2011), débutée avec la signature des accords de paix mettant fin à la guerre civile Nord/ Sud, n'a que partiellement ouvert les espoirs de reprise de ce chemin inclusif, car derrière les objectifs affichés de remplacer une ethnic identity par une civic identity, l'ancienne binarité a été consolidée (Bereketeab 2014). De manière concomitante, le conflit au Darfour, qui a émergé sur la scène médiatique en 2003 (Prunier 2005), et celui moins médiatisé dans l'est du pays (2005-2006), fournissaient une autre illustration de l'échec de la construction d'une identité nationale inclusive. Ainsi, la séparation du Soudan du Sud en juillet 2011 a permis au gouvernement de Khartoum d'affirmer avec plus de force encore le caractère incontestable d'un Soudan comme nation exclusivement arabe et musulmane (Woodward 2013). Les chercheurs n'ont pas manqué de saisir le tournant historique qui s'est imposé en 2011 et de s'interroger en conséquence sur les possibles redéfinitions des études soudanaises (Calkins, Ille \& Rottenburg 2015 ; Sharkey, Vezzadini \& SeriHersch 2015 ; Franck \& Vezzadini 2016). Tout en souscrivant au constat de la portée inédite de ce tournant, il nous a semblé important dans ce numéro de partir de données d'enquêtes menées au Soudan «post-2011» pour saisir la césure, sans pour autant négliger l'histoire plus profonde qui a imprégné les agencements de discours et de pratiques dans lesquels s'articulent de manière riche et singulière les catégories d'ethnicité, de religion, de nationalisme.

Dans cette perspective, si l'apport de l'anthropologie imprègne la problématique de ce numéro, celui de l'histoire y apparaît également incontournable. Ainsi, les micro-histoires (Bensa 1996) des groupes sociaux étudiés croisent l'histoire des formations étatiques nationales ou celles d'institutions religieuses à vocation transnationale. L'entrelacement des régimes de temporalité divers, celui entre différentes échelles spatiales, a attiré notre attention de chercheurs, nous rendant soucieux de ne pas limiter au «présent ethnographique » (dans un sens qui dépasse la seule logique grammaticale) la scène où se font et se défont les conceptualisations et articulations des catégories identitaires. Dans cette perspective, le dialogue avec nos collègues historiens a été fondamental. 
Nous avons déjà évoqué les articles d'Iris Seri-Hersch et d'Anaël Poussier, qui invitent, pour le premier, à revenir sur les politiques scolaires du tournant crucial entre fin de la colonisation et première phase de l'indépendance, pour le second, à remonter à la période mahdiste afin d'observer les stratégies de cet État précolonial dans la gestion des territoires et des identifications des populations. Tout aussi éclairant est le dialogue entre une politiste et une historienne, Florence Brisset-Foucault et Elena Vezzadini, autour du livre de cette dernière, Lost Nationalism. Revolution, Memory and Anti-colonial Resistance in Sudan (Vezzadini 2015) : dans cet épisode souvent oublié de la première révolution nationale qui date de 1924, on retrouve les traces d'une question nationale qui ne peut être comprise qu'en mettant au jour son rapport à l'esclavage ainsi que l'articulation complexe entre race et classe, identité ethno-tribale et affiliation religieuse.

La Révolution de «décembre» (2018-2019):

un tournant pour le complexe identitaire soudanais?

Alors que l'ambition d'une contextualisation historique se confirme comme féconde, dans son double objectif de renouer le fil entre courte et longue durée et de postuler le dynamisme intrinsèque des processus identitaires, la tentation de s'appuyer sur les événements d'un temps tout récent, d'une actualité encore en cours et en train de se configurer, offre un terrain plus glissant. Pourtant, si la troisième révolution soudanaise a émergé à un moment où la plupart des terrains et des réflexions à la base de ce projet éditorial étaient déjà en phase avancée, il nous a paru indispensable de saisir les échos établis par cette contingence inédite avec notre problématique. À ce titre, les premières protestations massives qui se sont déroulées au Soudan à partir du 19 décembre 2018 (Deshayes \& Vezzadini 2019) ont forcé notre attention. À cette occasion, le slogan partagé par les manifestants, "We are all Darfurians », qui s'opposait à la tentative de criminalisation du mouvement par le gouvernement selon des logiques récurrentes d'ethnicisation du conflit, nous a interpellés, tout comme le refus des personnes arrêtées de décliner leur appartenance tribale aux forces de police. La suite de ce mouvement révolutionnaire, jusqu'à sa victoire en avril 2019, puis dans les étapes de mise en place laborieuse d'une transition guidée par les civils, semble confirmer que ce moment de césure, en termes de formes de gouvernance politique du pays, constitue également une arène de négociation et de redéfinition de la place des trois catégories identitaires, ethnicité, religion, nationalisme. Le glossaire de la troisième révolution, présenté dans ce numéro par ses deux auteurs (Casciarri \& Manfredi 2020), revient sur les « significations situées » 
des slogans qui y ont émergé et qui sont, encore une fois, une démonstration de la manière dont les catégories d'ethnicité, de religion et de nationalisme s'articulent dans le temps (long et court) de l'histoire soudanaise. Dans cette même perspective, l'article de Mohamed Bakhit atteste de la reproduction de rapports différenciés au pouvoir qui s'inscrivent dans la morphologie de l'espace urbain de la capitale, où les déplacés internes, issus pour beaucoup des régions marginalisées et en conflit, ont été largement relégués en périphérie de la ville, voire hors de la ville. Sa démonstration s'attache à montrer que, depuis l'indépendance, les modalités de contestation s'articulent, d'une part autour d'une résistance armée dans les régions périphériques marginalisées qui n'a jusqu'ici pas abouti à des changements de pouvoir et, d'autre part, autour de contestations pacifistes menées au centre du pays et de la ville et portée par des populations du centre (arabo-musulmanes, awlad albalad $^{6}$ ) qui ont conduit à des changements de pouvoir en 1964, en 1985 et en 2019. Sa réflexion porte également sur les slogans inclusifs de la révolution, notamment celui déjà cité "We are all Darfourians », et sur l'ambivalence de leur réception par les populations concernées, dans un contexte où l'usage de la violence d'État ravive les tensions identitaires. La connotation interclassiste du soulèvement populaire, l'importance, du moins dans la phase initiale du mouvement, des villes de province, le glissement rapide d' «émeutes de la faim » en revendications politiques réclamant la chute d'un régime islamique doivent également nous interroger. C'est l'un des objectifs de l'article de Clément Deshayes qui nous livre une ethnographie fine de deux groupes d'opposition, de leur ancrage ethnique, social et politique à Khartoum comme de leur perception des « Autres » démontrant que leur idéal revendiqué n'est pas exempt de contradictions. S'il ne s'agit là que d'indices, que seule une observation plus prolongée des événements actuels pourrait resituer dans leur pleine signification, cela suggère néanmoins que la compréhension des enjeux complexes mobilisant au Soudan les catégories d'ethnicité, de religion et de nationalisme, mérite d'être affrontée dans ses intersections multiples, en rétablissant le lien entre les dynamiques de longue et de brève durée, et en osant aussi des incursions dans l'actualité des mouvements de changement radical. La relation entre transitions politiques, interventions étatiques dans le domaine de l'ethnicité et processus identitaires des communautés locales fait d'ailleurs l'objet de l'entretien réalisé par Francesco Staro avec

6. L'expression soudanaise «awlad al-balad» (littéralement «les enfants du pays ») est, à elle seule, une démonstration de la problématique de ce numéro, car elle désigne les populations du centre du pays, de la vallée du Nil et, plus particulièrement, les groupes arabes et musulmans qui, depuis l'indépendance (et du fait des dynamiques mises en place pendant la colonisation), captent, s'accaparent et conservent l'essentiel des ressources matérielles et politiques du pays. 
Günther Schlee, l'un des spécialistes majeurs des questions identitaires dans la Corne de l'Afrique : l'anthropologue nous livre ses réflexions sur leurs évolutions récentes en Éthiopie et au Soudan, suggérant ainsi l'importance de coupler la profondeur du regard spécifique sur une réalité nationale avec une comparaison plus large des mêmes dynamiques à l'échelle régionale.

Dans un ouvrage précédent portant sur les identifications mouvantes en Afrique de l'Est, Günther Schlee s'interrogeait déjà sur l'étrangeté de la success story du couplet dichotomique Arabes vs Africains, musulmans vs chrétiens, qui a pourtant prouvé depuis des décennies son inadéquation pour expliquer les réalités soudanaises (Schlee \& Watson 2009 : 12). Toujours au sujet des conflits labellisés comme identitaires qui ont ensanglanté la vie des Soudanais, d'autres chercheurs ont constaté avec regret les signes d'une répétition de l'histoire (Bleuchot, Delmet \& Hopwood 1991 : viii) et le « recyclage du passé » par une élite guidée par l'ethnocentrisme et la myopie politique (Harir 1994 : 16). Nous n'avons pas la prétention d'affirmer que la révolution soudanaise a définitivement surmonté la dimension binaire et hiérarchique des identités locales, ni que, grâce à elle, la triade ethnicitéreligion-nationalisme s'épanouira dans une nouvelle configuration à même d'éviter les pièges de l'essentialisation de ces catégories et son potentiel meurtrier. Bien plus modestement, nous espérons que ce numéro contribuera à renouveler le débat interdisciplinaire sur les processus identitaires en Afrique et donnera à voir les complexités actuelles et passées de la construction de ce « Nouveau Soudan $»^{7}$ que les acteurs de la dernière révolution s'efforcent de rebâtir en suivant l'idée d'un pluralisme identitaire comme source de richesse et moteur de justice.

Laboratoire architecture ville urbanisme environnement (LAVUE), Université Paris 8 Saint-Denis, France;

Pôle de recherche pour l'organisation et la diffusion de l'information géographique (PRODIG), Université Paris 1 Panthéon-Sorbonne, Paris, France;

Structure et dynamique des langues (SeDyL), CNRS, Villejuif, France;

Department of Sociology and Social anthropology, University of Khartoum, Soudan.

7. L'expression est empruntée à John Garang, leader de la rébellion sudiste qui portait un projet d'ambition nationale et inclusif, dit «New Sudan », un État séculier dans lequel la place de l'ensemble des populations marginalisées (et pas seulement celle des Sudistes) était renégociée. 


\section{BIBLIOGRAPHIE}

Abd Al-Rahim M., 1971, « Arabism, Africanism and Self-Identification in Sudan », in Y. Fadl Hasan (ed.), Sudan in Africa, Khartoum, Khartoum University Press : 228-239.

Abdelhay A., Makoni B. \& Makoni S., 2011, «The Naivasha Language Policy: The Language of Politics and the Politics of Language in Sudan », Language Policy, $10(1): 1-18$.

Abdelhay A., Abu Manga A. \& Miller C., 2015, «Language Planning and Policy in Sudan. From Local Vernaculars to National Languages », in B. CASCIARRI, M. A. ASSAL \& F. IRETON (eds.), Multidimensional Change in the Republic of Sudan (19892011). Reshaping Livelihoods, Conflicts and Identities, New York-Oxford, Berghahn Books : 263-280.

Ahmed A. M., 2002, Anthropology in the Sudan. Reflections by a Sudanese Anthropologist, Amsterdam, International Books.

Ambrosetti D., 2011, « Le Sud-Soudan en paix ? Sociologie politique d'une promesse d'indépendance », Dossier « Sud-soudan. Conquérir l'indépendance, négocier l'État », Politique africaine, 122 : 5-20.

Assal M. A., 2006, «Identity and Conflict over Natural Resources », Development, 43 : 101-105.

BARTH F. (ED.), 1969, Ethnic Groups and Boundaries, Oslo, Universitet Forlaget.

Baumann G., 1987, National Integration and Local Integrity. The Miri of the Nuba Mountains in Sudan, Oxford, Clarendon Press.

BENSA A., 1996, « De la micro-histoire vers une anthropologie critique », in J. REVEL (dir.), Jeux d'échelles. La micro-analyse à l'expérience, Paris, Gallimard-Le seuil : 37-70.

Bereketeab R., 2014, «Redefining National Identity and Nation-Building in Postsecession Sudans ; Civic and Ethnic Models ", Studies in Ethnicity and Nationalism, $14(2): 302-318$.

Bleuchot H., Delmet C. \& Hopwood D., 1991, «Introduction », in H. Bleuchot, C. Delmet \& D. Hopwood (eds.), Sudan. History, Identity, Ideology/Soudan. Histoire, identités, idéologies, London, Ithaca Press : vii-xvi.

Calkins S., Ille E. \& Rottenburg R. (eds.), 2015, Emerging Orders in Sudan, Mankon, Langaa Research \& Publishing. 
CAsciarri B., 2016, «Être, devenir et ne plus être janûbi. Parcours de l'identité "sudiste" entre le CPA et l'après 2011 dans un quartier populaire de la ville de Khartoum (Deim) », Égypte/Monde arabe, $3^{\mathrm{e}}$ série, $14: 65-84$.

— 2018, «Introduction. The Anthropology of Law in Muslim Sudan », in B. CASCIARRI \& M. A. BABIKer (eds.), Anthropology of Law in Muslim Sudan. Land, Courts and the Plurality of Practices, Leiden, Brill («Leiden Studies in Islam and Society ») : 1-19.

Casciarri B., Assal M. A. \& Ireton F. (eds.), 2015, Multidimensional Change in Sudan 1989-2011. Reshaping Livelihoods, Conflicts and Identities, New York-Oxford, Berghahn Books.

Casciarri B. \& Manfredi S., 2020, «Freedom, Peace and Justice. A Glossary of the Third Sudanese Revolution », Working Paper no. 2, Programme AUF, Arabité, islamité, « soudanité », <https://f-origin.hypotheses.org/wp-content/blogs.dir/1125/ files/2020/03/Working-Paper-2_Barbara-Casciarri-Stefano-Manfredi_A-Glossaryof-the-Third-Sudanese-Revolution_March-2020-1.pdf>.

Chevrillon-Guibert R., Bach J.-N. \& Franck A. (eds.), 2020, Dossier « Soudan : Jusqu'au bout du régime de l'Ingaz », Politique africaine, 158 (2).

Delmet C., 1991, « Société dominante et cultures locales. Violence et intégration au Dar Funj », in H. Bleuchot, C. Delmet \& D. Hopwood (eds.), Sudan. History, Identity, Ideology/Soudan. Histoire, identités, ideologies, London, Ithaca Press : 121-142.

Deshayes C. \& Vezzadini E., 2019, " "Quand le consensus se fissure”. Processus révolutionnaire et spatialisation du soulèvement soudanais», Politique africaine, $154: 149-178$.

Doornbos P., 1988, « On Becoming Sudanese », in T. BArnetT \& A. AbdelKarim (eds.), Sudan. State, Capital and Transformation, London, Croom Helm : 99-120.

Eberhard D. M., Simons G. F. \& Fennig C. D. (EDS.), 2020, Ethnologue: Languages of the World, 23 ${ }^{\text {th }}$ edition, Dallas, SIL International, <http://www.ethnologue.com>.

Fadl Hasan Y., 1967, The Arabs and the Sudan, Khartoum, Khartoum University Press.

Fluehr-Lobban C., 1987, Islamic Law and Society in Sudan, London, Franck Cass.

Franck A. \& VeZZAdini E. (EDS.), 2016, numéro spécial « Sudan, Five Years After the Independence of South Sudan », Égypte/Monde arabe, 14.

Grandin N., 1982, Le Soudan nilotique et l'administration britannique (1898-1956), Leiden, Brill.

HaAland G., 1969, «Economic Determinants in Ethnic Processes », in F. BARTH (ed.), Ethnic Groups and Boundaries, Oslo, Universitet Forlaget : 58-73.

— 2014, «Event Focused Fieldwork and Comparative Methodology : Exploring Ethnic Boundaries and Cultural Variation », in N. LosHINI (ed.), An Ethnography of Global Landscapes and Corridors, London, Intech open editions : 23-56. 
HARIR S., 1994, « Recycling the Past in the Sudan. An Overview of Political Decay », in S. Harir \& T. TVedt (eds.), Short-Cut to Decay. The Case of the Sudan, Uppsala, Nordiska Afrikan Institutet : 10-67.

Harir S. \& Tvedt T. (EDS.), 1994, Short-Cut to Decay. The Case of the Sudan, Uppsala, Nordiska Afrikan Institutet.

Hummel E., 2014, « Standing the Test of Time. Barth and Ethnicity », Coolabah, 13: 46-60.

MacMichael H. A., 1922, A History of the Arabs in Sudan, Oxford, Oxford University Press.

Mamdani M., 2012, Define and Rule. Native as Political Identity, London-Cambridge, Harvard University Press.

MANFREDi S., 2015, « One Tribe, One Language. Ethnolinguistic Identity and Language Revitalization among the Laggori in the Nuba Mountains », in B. CASCIARRI ET AL. (eds.), Multidimensional Change in Sudan 1989-2011, New York-Oxford, Berghan Books : 281-301.

Manger L., 2002, «Religion, Identities and Politics : Defining Muslim Discourses in the Nuba Mountans of the Sudan », Journal of Arabic and Islamic Studies, 4 (1-2) : 132-152.

- 2012, «Anthropological Reflections on the Break-up of Sudan », International Journal of Middle Eastern Studies, 44 : 327-329.

Mazrui A. A., 1971, «The Multiple Marginality of Sudan », in Y. Fadl Hasan (ed.), Sudan in Africa, Khartoum, Khartoum University Press : 240-255.

Miller C., 2006, «Languages, Identities and Ideologies : A New Era for the Sudan?», Proceedings of the 7th International Sudan Studies Conference, Bergen, University of Bergen : 149-174.

O'BrIEN J., 1986, « Through a Reconstitution of Ethnicity. Capitalist Expansion and Cultural Dynamics in Sudan », American Anthropologist, 88 (4) : 898-907.

Prunier G., 2005, Darfour. Un génocide ambigu, Paris, Éditions La Table Ronde.

SchleE G. \& Watson E. E. (EDS.), 2009, Changing Identifications and Alliances in NorthEast Africa, Vol. 3, Sudan, Uganda and the Ethiopia-Sudan Borderlines, New YorkOxford, Berghahn Books.

ScotT J. C., 1998, Seeing Like a State. How Certain Schemes to Improve the Human Condition Have Failed, New Haven, Yale University Press.

Seri-Hersch I., 2018, Enseigner l'histoire à l'heure de l'ébranlement colonial. Soudan, Égyte, empire britannique (1943-1960), Paris, Karthala-IISMM. 
Sharkey H. J., 2007, « Arab Identity and Ideology in Sudan : The Politics of Language, Ethnicity, and Race », African Affairs, 107 (426) : 21-43.

Sharkey H.J., Vezzadini E. \& Seri-Hersch I., 2015, « Rethinking Sudan Studies : a Post-2011 Manifesto », Canadian Journal of African Studies, 49 (1) : 1-18.

Sorbo G. \& Ahmed A. M., 2013, « Introduction. Sudan's Durable Disorder », in G. Sorbo \& A. M. Ahmed (eds.), Sudan Divided. Continuing Conflict in a Contested State, New York, Palgrave Macmillan.

Trimingham J. S., 1949, Islam in the Sudan, Oxford, Oxford University Press.

VeZZAdini E., 2015, Lost Nationalism : Revolution, Memory, and Anti-Colonial Resistance in Sudan, Woodbridge, Suffolk, James Currey.

WoOdWARD P., 2013, « Sudan after the South Secession : Issues of Identity », in G. M. SORBO \& A. M. Ahmed (eds.), Sudan Divided. Continuing Conflict in a Contested State, New York, Palgrave Macmillan : 89-102. 